\title{
Correlation of TAZ Immunohistochemistry Expression and Breast Carcinoma
}

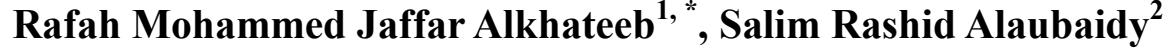 \\ ${ }^{1}$ Imamain Kadhymien Teaching Hospital, Baghdad, Iraq \\ ${ }^{2}$ Pathology Department \& Forensic Medicine, College of Medicine, University of Baghdad, Baghdad, Iraq \\ Email address: \\ rafahmalkhateeb@gmail.com(R. M. J. Alkhateeb),dr_salimhamoudi@yahoo.com(S. R. Alaubaidy) \\ ${ }^{*}$ Corresponding author
}

\section{To cite this article:}

Rafah Mohammed Jaffar Alkhateeb, Salim Rashid Alaubaidy. Correlation of TAZ Immunohistochemistry Expression and Breast Carcinoma. International Journal of Clinical Oncology and Cancer Research. Vol. 3, No. 1, 2018, pp. 10-13. doi: 10.11648/j.ijcocr.20180301.12

Received: March 6, 2018; Accepted: March 20, 2018; Published: April 12, 2018

\begin{abstract}
Overexpression of TAZ induces cell transformation and tumor-forming ability in mammary epithelial cells. The deactivation of the Hippo pathway, which leads to up regulation of YAP and TAZ is frequently observed in many human cancers. The study samples included seventy four formalin-fixed, paraffin embedded tissue blocks which have been diagnosed as forty four cases of breast carcinoma and their lymph nodes. In the present study from 74 total cases, 33cases (44.6\%) were showing TAZ marker positive while 41cases (55.4\%) were showing negative immunohistochemistry for TAZ marker. Sub classification of malignant cases, 11 malignant breast cancer cases (25\%) showed no TAZ expression, while 20 malignant cases $(45.5 \%)$ were strongly positive for TAZ expression. There is significant correlation between breast carcinoma grade, stage and TAZ expression.
\end{abstract}

Keywords: Breast Tumor, Breast Cancer, TAZ Expression

\section{Introduction}

Breast cancer is a family of diseases where cells in the breast tissue grow and divide without normal control. This growth of cells forms a mass or lump called a tumor. Tumors are either benign (not cancerous) or malignant (cancerous). [1]

In 1997 Dick et al. proposed that there is a certain subpopulation of cells in tumors that possess stem cell like properties.[2] These subpopulations where termed: cancer stem cells (CSC) or tumor-initiating cells. [3] CSC possesses self-renewal properties and can differentiate into daughter cells. CSC also has increased chemo-resistance, can promote metastasis and can survive treatment resulting in new tumors causing relapses. [2, 3]

TAZ (Transcriptional co-activator with PDZ-binding motif) contains one WW domain, which allows it to bind other partners that exhibit proline-rich modules named PY motifs.[4] In the cell nucleus TAZ binds with the primary partners which are TEA domain (TEAD) transcription factors. TAZ function as transcriptional co-activator for them. [5] Mammalian homologues of the proteins in the Hippo pathway have been characterized and grouped into three components: the upstream regulatory factors involved in cell-to-cell signaling (FRMD6, NF2), the kinase core (MST, WW45, LATS, MOB1), and the downstream transcriptional machinery (YAP, TAZ, TEAD), which leads to the expression of genes involved in cell proliferation and anti-apoptosis. [6] overexpression of TAZ induces cell transformation and tumor-forming ability in mammary epithelial cells.[7] The deactivation of the Hippo pathway, which leads to up regulation of YAP (Yes associated protein) and TAZ is frequently observed in many human cancers. The tumorigenic properties of TAZ may be due to WW and PDZ-binding domains, which play a crucial role in selfrenewal of mammary tissue. [8] WW domains of TAZ act through interaction with PPXY motifs of some transcriptional factors. For instance the Runx transcription factors (Runx1, Runx2 and Runx 3 that are involved in carcinogenesis and cancer metastasis. Both YAP and TAZ can potentiate Runx activity through WW-PPXY interaction.[9] Moreover, transduction of MCF10A cells with the WW domain mutation results in significantly fewer colonies in soft agar assay in comparison to the wild-type TAZ. [10] The highly conserved PDZ-binding motif localized in the C-terminus of TAZ also confers mammary tumor formation potential. It localizes TAZ into discrete nuclear 
foci and is essential for TAZ-stimulated gene transcription. [11] TAZ promotes breast cancer cell migration, invasion, and EMT (Epithelial-to-mesenchymal transition) predominately through interacting with TEADs.[12] The TAZ/TEAD complexes induce the transcription of AREG (Amphiregulin) to promote cell migration. Knockdown of AREG partially reduces the TAZdependent migration. Moreover, the expression of TAZ and AREG is positively correlated in breast cancer tissues. [13] The Hippo pathway plays an important role in drug resistance of breast cancer. [12] Taxol (paclitaxel) is a first-line chemotherapeutic drug used for breast cancer. TAZ is necessary for Taxol resistance in human breast cancer cells. [11] TAZ contributes to Taxol resistance by inducing the transcription of Cyr61 and CTGF. In addition, TAZ also causes the doxorubicin resistance in breast cancer cells. [12]

\section{Material and Method}

The study samples included seventy four formalin-fixed, paraffin embedded tissue blocks which have been diagnosed as forty four cases of breast carcinoma and their lymph nodes obtained from the department of Pathology/ oncology hospital /Medical City during the period from January 2014 to January 2016, twenty cases diagnosis as benign breast tissue(fibro adenoma) obtained from ALSHAHEED GHAZI TEACHING HOSPITAL /medical city during January2014 to January 2016 and ten cases of normal breast tissue obtained from forensic medical center.

The diagnosis of each case (breast carcinoma, corresponding lymph node and fibro adenoma (was confirmed by examining the hematoxylin and eosin (H\&E) sections for histopathological reevaluation by two specialized pathologists.

The fresh breast tissue from cadaver were chosen as a sample of ten normal breast tissue. Demographic and clinical data provided by the surgeon were obtained from the surgical pathology reports available with the tissue specimens, including patient's age, sex, clinical presentation, site of the tumor, tumor size, histological subtype, lymph node involvement, grading, and staging. Then we stain for immunohistochemistry marker TAZ, Positive controls For TAZ monoclonal antibody one tissue block of gall bladder was used. Negative control used for indicating the properness of the staining techniques, as positive control; one negative control was used for each set of test run.

\subsection{Principles of the Test}

Immunohistochemistry (IHC) enzyme labeled technique is a two-step indirect process, where the enzyme (peroxidase) is conjugated to a secondary reagent (link Antibody), providing an additional step for amplification of the antigen-antibody binding event. Biotin-Streptavidine amplified (B-SA) system is one of the most common linkers used in this method. A specific primary antibody will react with its corresponding antigen in the tissue, and then the biotin-labeled secondary antibody will bind to that primary $\mathrm{Ab}$. When the conjugate (Streptavidine bounded enzyme) is added, the biotinylated $\mathrm{Ab}$ will form a complex with the enzyme-conjugates streptavidin, and by adding the chromogene substrate, a colorimetric reaction will happen at the antigen binding site. The type of the chromogenic substrate depends on the type of the enzyme used. Thus, DAB (3, 3" diaminobenzidine) substrate offers the greatest sensitivity in the horseradish peroxidase enzyme system as a colorimetric chromogene, and a brownish precipitate will form at the antigen binding site.

\subsection{Evaluation of Immunohistochemistry Staining}

Brown nuclear and cytoplasmic reaction was regarded positive for TAZ. Immunohistochemical scoring was implemented by two independent observers who were blinded to the clinical data. the staining intensity of cells was classified into four intensity scores: [14]

No staining, 0; light staining, 1; moderate staining, 2; dark staining, 3 .

The percentage of positively-stained cells was classified into five grades with percentage scores as follows:10\% staining, $0 ; 10-25 \%$ staining, $1 ; 25-50 \%$ staining, $2 ; 50-75 \%$ staining, 3 ; and $>75 \%$ staining, 4 .

The staining positivity of FoxM1(Forkhead Box Protein M1), EPCAM (Epithelial cell adhesion molecule), CD47 (Cluster of differentiation 47) and TAZ was calculated using the following formula: Total score $=$ intensity score $\times$ percentage score.

Based on the total score of each tissue, results of Negative $\leq 3$, Weakly positive $>3$ to $\leq 6$ Strong positive $>6$

\section{Result}

\subsection{TAZ Expression}

In the present study from 74 total cases, 33 cases $(44.6 \%)$ were showing TAZ marker positive while 41 cases $(55.4 \%)$ were showing negative immunohistochemistry for TAZ marker as shown in below figure 1

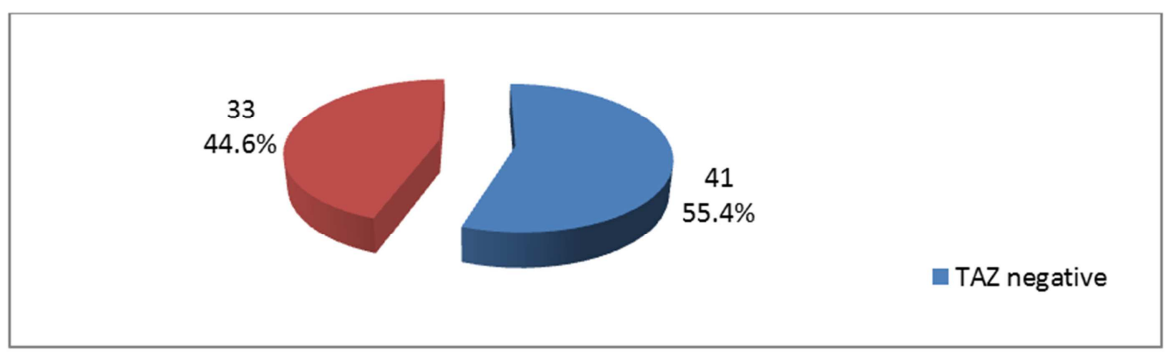

Figure 1. A pie chart was shown distribution of cases according to TAZ marker immunohistochemistry staining. 
Sub classification of malignant cases, 11 malignant breast cancer cases (25\%) showed no TAZ expression, while 20 malignant cases $(45.5 \%)$ were strongly positive for TAZ expression. As shown in the figure below.

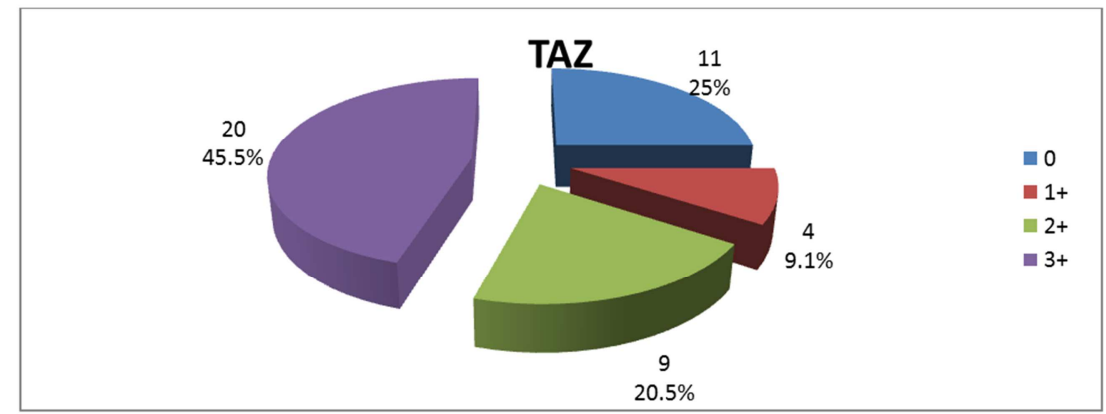

Figure 2. A pie chart was showing classification of malignant cases according to TAZ immunohistochemistry staining.

Table 1. A table showing the significant correlation between TAZ expression and the grade of breast carcinoma cases. *: significant correlation, NS: not significant.

\begin{tabular}{|c|c|c|c|c|c|c|c|c|c|c|}
\hline & & count & $\%$ & count & TAZ \% & Count & $\%$ & Count & $\%$ & P value \\
\hline \multirow{3}{*}{ Grade } & I & 1 & $9.1 \%$ & 2 & $50.0 \%$ & 0 & $.0 \%$ & 0 & $.0 \%$ & \multirow{3}{*}{$\begin{array}{l}0.0005 \\
*\end{array}$} \\
\hline & II & 10 & $90.9 \%$ & 1 & $25.0 \%$ & 6 & $66.7 \%$ & 8 & $40.0 \%$ & \\
\hline & III & 0 & $.0 \%$ & 1 & $25.0 \%$ & 3 & $33.3 \%$ & 12 & $60.0 \%$ & \\
\hline
\end{tabular}

Table 2. A table showing the significant correlation between TAZ expression and the stage of breast carcinoma cases. *: significant correlation, NS: not significant

\begin{tabular}{|c|c|c|c|c|c|c|c|c|c|c|}
\hline Stage & & Count & $\%$ & TAZ Count & $\%$ & Count & $\%$ & Count & $\%$ & P value \\
\hline & I & 0 & $.0 \%$ & 1 & $25.0 \%$ & 0 & $.0 \%$ & 0 & $.0 \%$ & 0.014 \\
\hline & IIA & 3 & $27.3 \%$ & 2 & $50.0 \%$ & 1 & $11.1 \%$ & 1 & $5.0 \%$ & $*$ \\
\hline & IIB & 8 & $72.7 \%$ & 1 & $25.0 \%$ & 4 & $44.4 \%$ & 6 & $30.0 \%$ & \\
\hline & IIIA & 0 & $.0 \%$ & 0 & $.0 \%$ & 2 & $22.2 \%$ & 7 & $35.0 \%$ & \\
\hline & IIIB & 0 & $.0 \%$ & 0 & $.0 \%$ & 0 & $.0 \%$ & 2 & $10.0 \%$ & \\
\hline & IIIC & 0 & $.0 \%$ & 0 & $.0 \%$ & 2 & $22.2 \%$ & 4 & $20.0 \%$ & \\
\hline
\end{tabular}

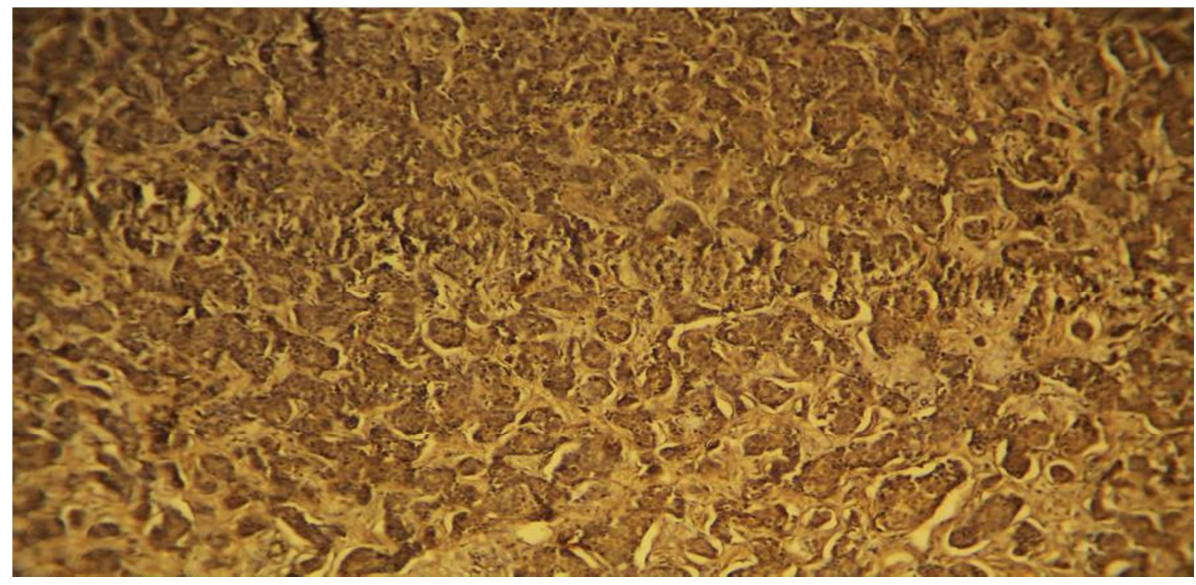

Figure 3. Breast carcinoma case show strong expression of TAZ immunohistochemistry marker.

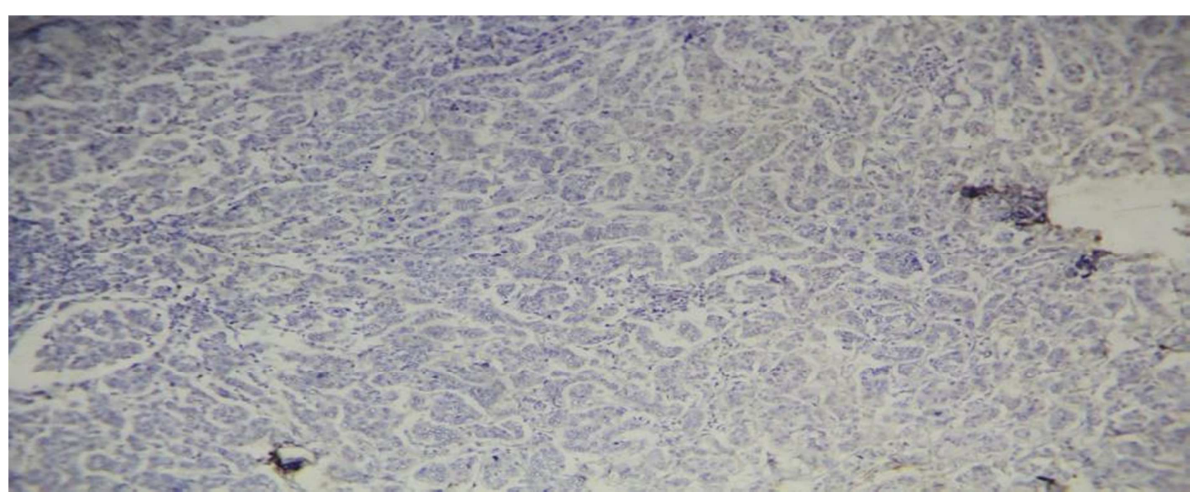

Figure 4. The slide was showing breast carcinoma case with negative expression to TAZ immunohistochemistry marker. 


\section{Discussion}

In the present analysis TAZ is overexpressed in $44.6 \%$ of breast cancer samples, twelve cases $(60 \%)$ of poorly differentiated breast carcinoma were showing strong expression of immunohistochemistry TAZ marker while the well differentiated breast carcinoma cases showed weak expression of immunohistochemistry TAZ marker (all well differentiated cases showed either no TAZ or weak expression ), Aleksandra Glapa1, Aleksandra Nijak [15] has previously find the same result, CSCs are indeed enriched in high-grade breast cancers. That fact suggests that TAZ plays a critical role in the migration, invasion, and tumorigenesis of breast cancer cells, In this ongoing study, it have been noticed the higher expression levels of TAZ in more invasive breast cancer cells. This observation prompted us to investigate the physiologic/clinical relevance and the role of $\mathrm{TAZ}$ in the tumorigenesis of breast cancer.

In present study all fibro adenoma cases and normal breast tissue were showing no expression for TAZ immunohistochemistry marker, so it was normal to find no TAZ expression in normal tissue and no role of TAZ in benign breast tumor fibro adenoma

Also this study discovered significant correlation between TAZ immunohistochemistry and stage of breast carcinoma it was found that all stage III carcinoma showed strong expression of TAZ immunohistochemistry marker either score III or score II while stage I breast carcinoma cases were showing weak expression of TAZ immunohistochemistry marker score I. Marcello Maugeri-Saccà a, b, Ruggero De Maria agree with this study finding's [16].

\section{Conclusion}

It is worthy to consider TAZ as a predictive and prognostic factor for breast carcinoma. With strong TAZ immunohistochemistry expression there will be an increase in the probability for breast carcinoma to be more aggressive, high grade, larger size, and increase the susceptibility for its metastasis.

\section{References}

[1] Susan G. Komen. What is breast cancer? Fact for life. 2016; $2: 16$.

[2] Han L, Shi S, Gong T, Zhang Z, Sun X. Cancer stem cells: therapeutic implications and perspectives in cancer therapy. Acta Pharmaceutica Sinica. B 2013; 3: 65-75.

[3] Takahashi R, Takeshita F, Fujiwara T, Ono M, Ochiya T. Cancer stem cells in breast cancer. Cancers. 2011; 3: 13111328.
[4] Sudol M, Sliwa K, Russo T. Functions of WW domains in the nucleus. FEBS Lett. Feb. 2001; 490(3):190-195.

[5] Chan SW, Lim CJ, Guo K, Ng CP, Lee I, Hunziker W, Zeng Q, Hong W. A role for TAZ in migration, invasion, and tumorigenesis of breast cancer cells. Cancer Res. Apr. 2008; 68(8):2592-2598.

[6] Hung Yi Kristal Kaan1, Siew Wee Chan1, Siew Kim Joyce Tan1, Fusheng Guo1, Chun Jye Lim1, Wanjin Hong1 \& Haiwei Song"Crystal structure of TAZ-TEADcomplex reveals a distinctinteraction mode from that of YAPTEAD complex", scientific reports. 2017 may.

[7] D. B. SeligsonEpithelial cell adhesion molecule (KSA) expression: pathobiology and its role as an independent predictor of survival in renal cell carcinoma Clin. Cancer Res., 10 (2004), pp. 2659-2669.

[8] Laoukili, J., Alvarez-Fernandez, M., Stahl, M., and Medema, R. H. FoxM1 is degraded at mitotic exit in a Cdh1-dependent manner. Cell Cycle 7.2008b. 2720-2726.

[9] Anders, L., Ke, N., Hydbring, P., Choi, Y. J., Widlund, H. R., Chick, J. M., et al. A systematic screen for CDK4/6 substrates links FOXM1 phosphorylation to senescence suppression in cancer cells. Cancer Cell. 2011.20, 620-634.

[10] Wang, I. C., Chen, Y. J., Hughes, D., Petrovic, V., Major, M. L., Park, H. J., et al. Forkhead box M1 regulates the transcriptional network of genes essential for mitotic progression and genes encoding the SCF (Skp2-Cks1) ubiquitin ligase. Mol. Cell. Biol. 2005.25, 10875-10894.

[11] Laoukili, J., Kooistra, M. R., Bras, A., Kauw, J., Kerkhoven, R. M., Morrison, A., et al. FoxM1 is required for execution of the mitotic programme and chromosome stability. Nat. Cell Biol. 2005.7, 126-136.

[12] Singh, B., Gogineni, S. K., Sacks, P. G., Shaha, A. R., Shah, J. P., Stoffel, A., et al. Molecular cytogenetic characterization of head and neck squamous cell carcinoma and refinement of $3 q$ amplification. Cancer Res.2010. 61, 4506-4513.

[13] Pilarsky, C., Wenzig, M., Specht, T., Saeger, H. D., and Grutzmann, R. Identification and validation of commonly overexpressed genes in solid tumors by comparison of microarray data. Neoplasia 2004.6, 744-750.

[14] Jing Zhang, Kundong ZhangLisheng Zhou, Weidong Wu, Tao Jiang, Jun Cao, Kejian, Huan, Zhengjun Qiu, Chen Huang. Expression and potential correlation among Forkhead box protein M1, Caveolin-1 and E-cadherin in colorectal cancer. oncology letters. July 28, 2016.

[15] Aleksandra Glapa1, Aleksandra Nijak. TAZ oncogene as a prognostic factorin breast cancer. Journal of Medical Science.2015. 2 (84).

[16] Marcello Maugeri-Saccà a, b, Ruggero De Maria. Hippo pathway and breast cancer stem cells. Critical Reviews in Oncology/Hematology 99 (2016) 115-122. 